\title{
Liposomal amphotericin $b$ and delayed presentation of renal tubular acidosis: a case report
}

\begin{abstract}
Background: Conventional form of Amphotericin B has been associated with renal tubular acidosis, decreased renal function and hypokalemia. Liposomal Amphotericin B (AmBisome) is suggested to be associated with less nephrotoxicity. We present a case of AmBisome associated kidney injury with delayed onset of renal tubular acidosis.
\end{abstract}

Keywords: liposomal amphotericin b, ambisome, nephrotoxicity, renal tubular acidosis

\author{
Volume 7 Issue I - 2017 \\ Muhammad Ameen, Naeem Abbas, Nasr \\ Rabih \\ Bronx-Lebanon Hospital Center, USA \\ Correspondence: Muhammad Ameen, Bronx-Lebanon \\ Hospital Center, 1650 Grand Concourse, Bronx, USA, \\ Email drmameenI5I@gmail.com
}

Received: July 7, 2017 | Published: July 17, 2017

\section{Case report}

26years old patient with medical history of HIV (CD4 count $<20$ and viral load $>54,000$ ) was admitted with severe frontal headache of 10days. After having a negative CT scan for SOL and raised ICP, LP was done and CSF analysis was suggestive of cryptococcal meningitis. Patient received Liposomal formulation of Amphotericin $\mathrm{B}$ and Flu cytosine for 2 weeks in the hospital. Patient was found to have persistent hypokalemia with the lowest nadir value of $2.6 \mathrm{mEq} / \mathrm{L}$ (Lab normal $3.5-5.0 \mathrm{mEq} / \mathrm{L}$ ) on day 9 of receiving Liposomal Amphotericin B and serum creatinine (S.Cr) worsened from $0.7 \mathrm{mg} / \mathrm{dl}$ to $1.5 \mathrm{mg} / \mathrm{dl}$ at the time of discharge (Lab normal for S.Cr $0.5-1.5 \mathrm{mg}$ / dl). He was discharged on Fluconazole $400 \mathrm{mg}$ once a day. One month later, patient came back with complaints of severe generalized body aches and weakness. At the time of admission, he was found to have potassium level of $1.7 \mathrm{mEq} / \mathrm{L}$, lowest serum potassium level on the day of admission was $1.3 \mathrm{mEq} / \mathrm{L}$ and serum creatinine $1.3 \mathrm{mg} / \mathrm{dl}$. Anion gap was 14. Spot urinalysis showed urine Ph of 7.0 (Lab Normal 6.008.00). 24-hour urine collection showed urine potassium $27.4 \mathrm{mEq} / \mathrm{L}$ (Lab normal $22-160 \mathrm{mEq} / \mathrm{L}$ ) and urine creatinine $57 \mathrm{mg} / \mathrm{dl}$ (Lab normal $20-320 \mathrm{mg} / \mathrm{dl}$ ), Urine potassium-creatinine ratio was 26.9. Patient was given intravenous potassium supplement and his symptoms improved significantly. He was started on oral potassium chloride and sodium bicarbonate supplementation, observed clinically and on labs, remained stable and was discharged home with K supplements. The impression was renal tubular acidosis type I secondary to recent AmBisome use. There was documented evidence of normal anion gap metabolic acidosis, renal wasting of potassium and hypokalemia in the setting of recent AmBisome.

\section{Discussion}

Conventional form of Amphotericin B has been associated with renal tubular acidosis, decreased renal function and hypokalemia. ${ }^{1}$ The exact mechanism of amphotericin B induced nephrotoxicity is unclear; it can lead to renal damage either through vasoconstriction, increasing cell membrane permeability through insertion into cell membranes, or through doxycholate, a detergent used as a solubilizing agent for amphotericin., ${ }^{2,3}$ Amphotericin B can also cause significant hypokalemia by altering the permeability of distal renal tubular cells, leading to an increase in the passive fluxes of potassium down it electrochemical gradient. ${ }^{4}$ Liposomal formulation of Amphotericin
B has been proposed to be associated with less renal toxicity. ${ }^{5,6}$ Liposomes are small vesicles consisting of phospholipid bilayer, containing Amphotericin. It can be preferentially taken by the reticuloendothelial system and macrophages, and thereby possibly enhanced therapeutic index and less toxicity. Leenders et al., ${ }^{7}$ compared liposomal amphotericin B with conventional amphotericin B formulation in the treatment of AIDS associated cryptococcal meningitis and showed that patients undergoing AmBisome treatment suffered significantly less nephrotoxicity compared with conventional Amphotericin B formulation (11.5\% vs. 40.7\%, p<0.05). ${ }^{7}$ In our case, patient suffered from decreased GFR, rise in S. Cr and hypokalemia after AmBisome use. AmBisome has been known to cause both significant hypokalemia and decreased renal function that was presumed to be the cause in our patient. The AmBisome related nephrotoxicity can present right after the start of medication or it may be delayed and can persist for months ${ }^{1}$ as in our case. The cause of this delayed presentation is unknown. The physicians must be aware of the possible delayed presentation of AmBisome induced nephrotoxicity and must monitor patient renal profile after giving AmBisome to their patients.

\section{Acknowledgements}

None.

\section{Conflicts of interest}

The authors report no conflict of interest.

\section{References}

1. Sawaya BP, Briggs JP, Schnermann J. Amphotericin B nephrotoxicity: the adverse consequences of altered membrane properties. J Am Soc Nephrol. 1995;6(2):154-164.

2.Heyman SN, Stillman IE, Brezis M, et al. Chronic amphotericin nephropathy: morphometric, electron microscopic, and functional studies. J Am Soc Nephrol. 1993;4(1):69-80.

3. Zager RA, Bredl CR, Schimpf BA. Direct amphotericin B-mediated tubular toxicity: assessments of selected cytoprotective agents. Kidney Int. 1992;41(6):1588-1594.

4. Gerkens JF, Branch RA. The influence of sodium status and furosemide on canine acute amphotericin B nephrotoxicity. J Pharmacol Exp Ther. 1980;214(2):306-311. 
5. Messori A, Fadda V, Maratea D, et al. Nephrotoxicity of different formulations of amphotericin B: summarizing evidence by network meta-analysis. Clin Infect Dis. 2013;57(12):1783-1784.

6. Falci DR, da Rosa FB, Pasqualotto AC. Comparison of nephrotoxicity associated to different lipid formulations of amphotericin B:a real-life study. Mycoses. 2015;58(2):104-112.
7. Leenders AC, Reiss P, Portegies P, et al. Liposomal amphotericin B (AmBisome) compared with amphotericin B both followed by oral fluconazole in the treatment of AIDS-associated cryptococcal meningitis. Aids. 1997;11(12):1463-1471. 